\section{Tolerance to kidney allograft transplanted into Type I diabetic patients persists after in vivo challenge with pancreatic islet allografts that express repeated mismatches}

\section{Dear Sir,}

Allogeneic islet transplantation can restore insulin production in C peptide negative patients with Type I (insulin-dependent) diabetes mellitus $[1,2]$. We recently reported three cases of surviving islet allografts, implanted in Type I diabetic patients under maintenance immune suppression for a kidney, transplanted years before [3]. Immunological studies indicated that islet graft survival was accompanied by low T-cell auto-reactivity and alloreactivity [4]. Other Type I diabetic patients $(n=4)$ with rapid islet allograft loss, showed high levels of islet specific autoreactivity, allospecific cytotoxic T-cells towards the HLA mismatches of the islet grafts, or both types of reactivity. Because islet allografts were composed of multiple pancreas donors, we were concerned that shared HLA antigens between the islet allografts and the kidney might jeopardise the function of the latter. Indeed, we could show the immunogenic potential of islet allografts, as they were able to induce vigorous and specific cytotoxic T-cell responses towards the mismatched HLA antigens (Fig. 1A, C). In all cases control cytotoxic T-cell responses towards autologous and third-party antigens were negative. Surprisingly, implantation of islet allografts that shared antigens with the kidney graft never resulted, however, in alloreactivity towards the repeated HLA mismatches (Fig.1B, D). These findings were corroborated by the lack of kidney graft rejection episodes in all patients, regardless of rapid islet allograft failure in some patients.

The low alloreactivity against shared antigens was furthermore characterised by the absence of an induction peak (Fig.1B, D) but a transient rise in cytotoxic T-cell precursor frequency towards novel-mismatched allodeterminants was observed. Even in case of successful islet grafts a clear but short-lived increase in islet specific alloreactivity towards novel mismatches was seen (Fig.1A). Our results strongly suggest that the kidney transplant is able to specifically reduce the immune response of the patient towards those islet antigens that are repeatedly mismatched antigens of the kidney. Frequently, high reactivity was measured towards novel mismatches, present on the same islet allograft as repeated mismatches. Our data support a role for the kidney graft in both the induction and maintenance of tolerance. In vivo rechallenge of the patients by the islet grafting did not break this tolerance whereas the response towards the novel-antigens remained unaffected, which illustrates the specificity of the tolerance.

Yours sincerely,

I. Stobbe, G. Duinkerken, J.J. van Rood, A. Lernmark, B.Keymeulen, D. Pipeleers, R.R.P. De Vries, F.H.J. Claas, B. O. Roep

\section{References}

1. Sharp DW, Lacy PE, Santiago JV, McCullough CS, Weide LG, Falqui L (1990) Insulin independence after islet transplantation into Type I diabetic patient. Diabetes 39: 515-518.

2. Eisenbarth GS, Stegall MD (1996) Islet and pancreas transplantation - autoimmunity and alloimmunity. N Eng J Med 335: 888-890

3. Keymeulen B, Ling Z, Gorus FK et al. (1998) Implantation of standardised beta-cell grafts in a liver segment of IDDM patients:graft and recipient characteristics in two cases of insulin-independence under maintenance immunosuppression for prior kidney graft. Diabetologia 41: 452-459

4. Roep BO, Stobbe I, Duinkerken G et al. (1999) Auto- and alloimmune reactivity to human islet allografts transplanted to insulin-dependent diabetes mellitus patients. Diabetes 48: $484-490$
Corresponding author: Dr. B. O. Roep, Department of Immunohaematology and Blood Bank, Leiden University Medical Center, Bldg 1 E3-Q, P.O. Box 9600, 2300 RC Leiden, The Netherlands 
Successful Islet transplantations
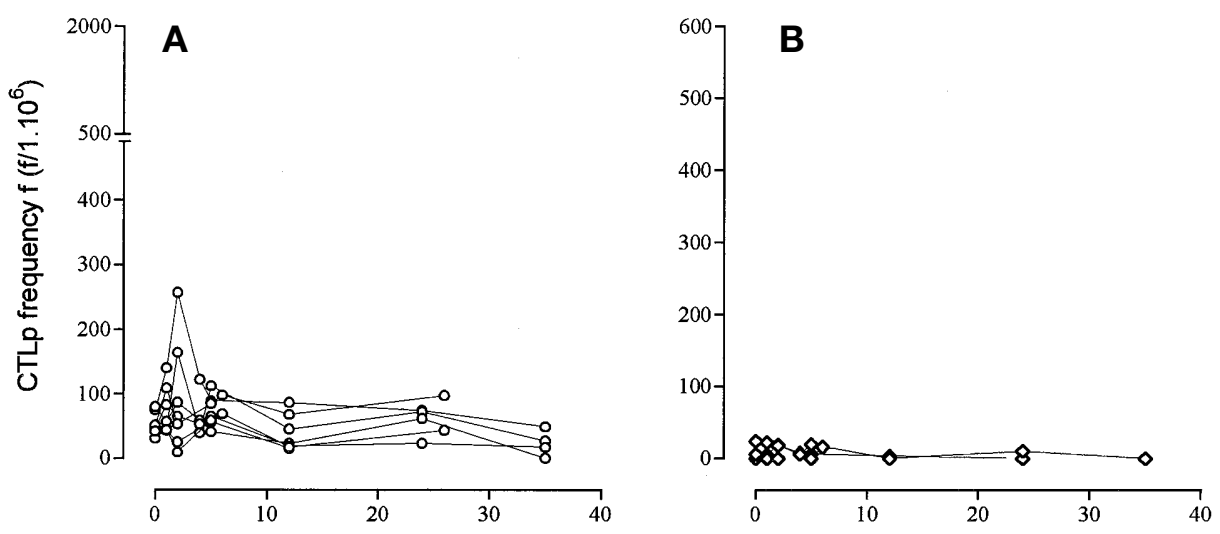

Unsuccessful Islet transplantations

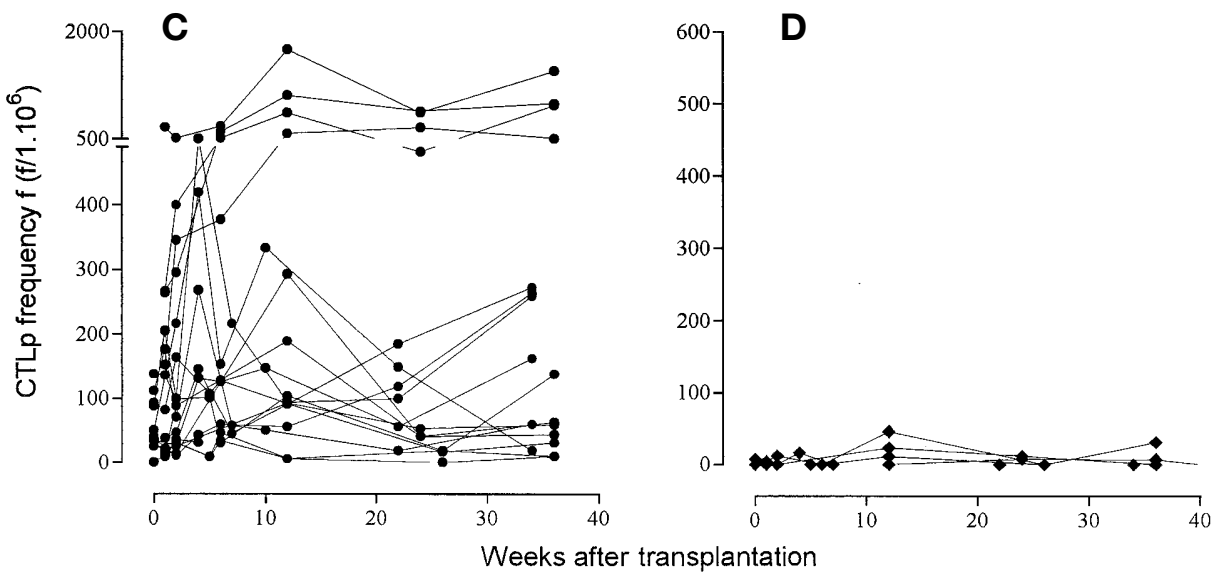

Fig.1A-D. Precursor frequencies of alloreactive cytotoxic Tcells (CTLp; expressed as absolute numbers per million peripheral blood nonnuclear cells) in patients successfully receiving islet allografts (i.e. persistent beta-cell function; $\mathbf{A}(\bigcirc)$, and $\mathbf{B}(\diamond)$, open symbols) and patients with failing islet allograft implants $(\mathbf{C}(\mathbf{O})$ and $\mathbf{D}(\diamond)$, closed symbols). Graphs $\mathbf{A}$ and $\mathbf{C}$ display CTLp frequencies against all mismatched HLA antigens expressed by the islet allografts in time (multiple targets per patient). Graphs $\mathbf{B}$ and $\mathbf{D}$ present frequencies of CTLp specific for HLA mismatches shared between the kidney and islet allografts (repeated mismatches). Detailed description of recipients, and kidney and islet grafts are published elsewhere [3, 4] 